\title{
Eine Schule führen in Zeiten der Krise
}

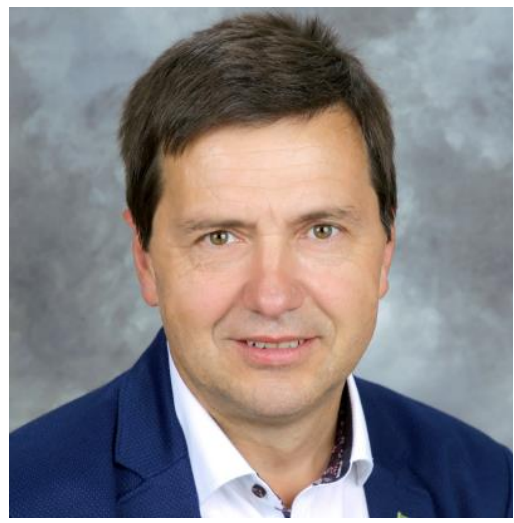

Foto: Starfoto
Eine Schule zu managen war auch schon vor der Pandemie eine Herausforderung. Nun wurde diese Aufgabe eine besondere. Dipl.-Ing. Martin Pfeffel trägt seit November 2017 die Verantwortung für Niederösterreichs zweitgrößte Schule, die HTL St. Pölten. Er versucht auch in schwierigen Zeiten, allen Anforderungen gerecht zu werden und Lösungen zu finden. Was das im schulischen Alltag bedeutet, beschreibt er uns im folgenden Interview.

Sie kommen aus der Wirtschaft, ihre Kernkompetenz ist die Fertigungstechnik. Wie geht es Ihnen im Schulalltag?

Martin Pfeffel: In den ersten beiden Jahren konnten mein Team und ich sehr viel umsetzen, sowohl im Umgang mit Industrie 4.0 als auch mit der neuen Oberstufe. Außerdem haben wir schon vor der COVID-19-Pandemie eine Gigabit-Glasfaserleitung installiert und waren somit für die zunehmende Digitalisierung bestens vorbereitet, ein glücklicher Zufall oder Weitblick. Die Schüler*innenzahlen wachsen stetig.

\section{Und in Bezug auf die COVID-19-Pandemie?}

Die Herausforderungen, die wir jetzt haben, hat uns wirklich keiner vorhergesagt beziehungsweise wurden wir dahingehend nicht geschult. In der Pandemiesituation sehen wir deutlich, dass eine funktionierende Kommunikation aller Beteiligten der entscheidende Punkt ist. Wenn wir auf die Pressekonferenzen, die im Prinzip bei Veränderungen der Maßnahmen am Beginn stehen, blicken, mussten wir erkennen, dass sie rechtlich mehr oder weniger unverbindlich waren, auch die Informationen, die vom Bildungsministerium an die Direktionen versandt wurden. Die rechtlich verbindlichen Informationen, die über einen 
Umweg - vom Bildungsministerium zur Bildungsdirektion und dann zu den regionalen Stellen - geschickt wurden, kamen leider oftmals sehr spät.

\section{Sie mussten oftmals instinktiv entscheiden?}

Es gab auch die eine oder andere Entscheidung, die man aus dem Bauch heraus schulautonom treffen musste. So wurde bei Pressekonferenzen im Moment der Entscheidungsfindung oftmals die einzige Richtlinie, die Sachlage, anders kommuniziert, als dann im speziellen Erlass definiert wurde. Natürlich können da manche Entscheidungen nicht immer perfekt sein.

\section{Kommunikation erwähnten Sie schon vorhin als wichtigen Punkt.}

Kommunikation ist nach wie vor ein Riesenthema und auch die Digitalisierung, die ja schon vor der Pandemie zum Teil umgesetzt wurde, fordert uns ständig. Wir in der HTL können uns nicht beklagen, da wir, wie gesagt, nach meiner Bestellung zum Direktor des Hauses, einige dieser Aufgaben schon vor der Pandemie erledigt haben. Sich gut aufzustellen, war für mich gleich zu Beginn ein sehr wichtiges Thema. Was an zusätzlichen digitalen Dingen bleibt, wie etwa Leihgeräte für Schüler*innen oder Untis Messenger, die während dieser schwierigen Situation zur Nutzung angeboten wurden, werden wir danach diskutieren müssen.

\section{Was zeigte sich als nützliches Tool?}

Die Plattform eduFLOW als digitalisiertes Kommunikationstool erwies sich als hilfreich. Die gesamte schulische Kommunikation mit Schüler*innen, Schulpersonal und speziell mit den Eltern - das sind bei uns ca. 4.000 Menschen - war dadurch leichter, besonders die automatisierte Rücksammlung der Antworten erleichtert die Administration im Schulalltag. Anders wäre es zurzeit aufgrund der wöchentlichen Information an die Eltern ein Ding der Unmöglichkeit. In unserem Bereich ist es sicher einfacher als in anderen Schulformen, Eltern zu „erziehen“, eine E-Mail-Adresse, die sie auch regelmäßig benützen, ihr Eigen zu nennen. Die digitalisierte Kommunikation erspart uns auch Portokosten.

\section{Wurden die Entscheidungen von den Eltern mitgetragen?}

In dieser Krise ist vieles zum Vorschein gekommen. Es ist natürlich gut, dass wir in einem demokratischen Staat leben und alle ihre persönliche Meinung äußern können, aber wenn es rechtlich verbindliche Erlässe gibt, die etwa die Maskenpflicht oder andere Maßnahmen reglementieren, und man das an die Schulgemeinschaft kommuniziert, müssen sich alle daran halten. Bei Schüler*innen und Lehrer*innen war das weniger das Problem, aber einige Eltern haben da doch merkwürdig reagiert.

\section{Was erlebt man da so als Direktor einer großen Schule?}

Als Direktor musste ich jedes Wochenende einen Brief an die Eltern per eduFLOW zur aktuellen Lage aussenden. Meist am Sonntag, weil sich die Situation von Freitag an ja immer änderte. Am Montag waren dann zehn bis 20 E-Mails von Eltern im Postfach, die durchaus 
staatsverweigernde Aussagen beinhaltet haben - von Querdenkenden, Maskenverweigernden bis hin zur Aufforderung, dass ich als Direktor eine Bestätigung ausfüllen sollte, dass ihr Kind nicht geimpft wird. Man glaubt es kaum, mit welch obskuren Dingen man als Schulleiter*in konfrontiert wird. Da ist es von entscheidender Bedeutung, dass man die Sicherheit hat, dass hinter den Informationen, die man ausgibt, auch Erlässe stehen, die rechtlich in Ordnung sind.

\section{Wie wirken Sie den oben genannten Wünschen entgegen?}

Eltern, die mit diesen Meinungen per E-Mail auf mich zukommen, rufe ich zumeist an. Danach bleiben ein, zwei Eltern über, die nicht überzeugt werden können, da nützt auch das persönliche Gespräch nichts. Ärgerlich ist, dass viele Eltern aus dem Internet vorgefertigte Formulare von sogenannten Expert*innen auf dem Gebiet der Juristerei verwenden, um die Schule, Lehrer*innen und die Direktion mit angedrohten Anzeigen unter Druck zu setzen.

\section{Stößt Ihnen in der aktuellen Diskussion noch etwas auf?}

Es gibt im schulischen Bereich zwei Fraktionen: Die eine denkt im Volksschul-, die andere im AHS-Schema. Die Thematik Matura wird zumeist auf die AHS-Matura reduziert, darunter leiden die berufsbildenden Schulen. Durch unsere fachpraktische Ausbildung sind wir meistens die Ausnahme, bei uns ist vieles anders als nach außen thematisiert wird. So ist etwa die aktuelle Einteilung - zwei Tage Präsenzunterricht, dann zwei Tage Home-Schooling - bei uns anders gestaltet. Wenn es beispielsweise um den Abstand in den Klassenräumen geht: Bei uns sind die Schüler*innen eine ganze Woche in Präsenz in der Schule, dann sind sie eine Woche im Home-Office, das stärkt die Klassengemeinschaft, die durch einen Schichtbetrieb leiden würde.

\section{Bleibt für andere Projekte Zeit?}

Große Schulentwicklungsprojekte, wie die semestrierte Oberstufe (SOST) oder das neue Qualitätsmanagementsystem (QMS), sind in den Hintergrund geraten. Jetzt tut sich wieder was, aber wenn ich das in dieser Zeit an meine Lehrer*innen kommuniziere, dann wird das eine Frustabladeplattform. Mit meinem Führungsteam bin ich im ständigen Kontakt, komplexe Fragen, wie die provisorische Lehrfächerverteilung und das Schulbudget, müssen bearbeitet werden. Strategische Projekte zu planen ist zurzeit eher schwierig. Veranstaltungen, wie zum Beispiel den Schulball, gibt es keine. Alle Dinge, die Spaß machen, wie Sommersportwoche oder Wintersportwoche, können nicht abgehalten werden. Auch die Pflege unserer Außenbeziehungen zu Firmen ist mühsamer geworden.

\section{Wie läuft die Kommunikation über Onlinekanäle?}

Jede Besprechung findet bei uns über die Plattform Microsoft Teams statt. Natürlich können die Informationen nicht so nachhaltig vermittelt werden wie bei Präsenzveranstaltungen. Seit März vergangenen Jahres musste ich fast jedes Wochenende für die Schule da sein. Unser erster positiver COVID-19-Fall ist auch an einem Freitag aufgetreten. Ständig ändern sich die 
Meldungsanforderungen an die Schulbehörde, oftmals müssen wir redundante Daten mit häufig wechselnden Tools senden. Im QMS hört man immer, dass man schulautonom herausfinden soll, welche Dinge uns weiterbringen und welche nicht. Leider ist es uns auch in Zeiten der Digitalisierung noch immer nicht möglich, dass wir auf das Schülerstammdatenblatt der Volkschule bis zum Ende der Schulkarriere zurückgreifen können, obwohl wir (fast) alle mit Sokrates arbeiten.

\section{Brachte die Krise noch neue Erkenntnisse?}

Durch den Ausfall der Tage der offenen Tür und dadurch, dass keine Schüler*innen schnuppern kommen konnten, wurden wir gezwungen, viel mehr in die sozialen Medien zu gehen. Vielleicht sehen wir in Zukunft dem Stress rund um diese Events gelassener entgegen. Auch die digitalisierte, webbasierte Organisation der Anmeldungen zeigte sich als positiv. Unser Sekretariat musste in Präsenz nur den Stempel draufgeben, so konnten wir im Zehnminutentakt 450 Leute durchschleusen. Unsere zukünftigen Schüler*innen kommen zurzeit mit sehr schönen Zeugnissen zu uns, die teilweise der COVID-19-Pandemie geschuldet sein könnten. Da werden wir schauen, was nächstes Jahr passiert.

\section{Wie begegnen Sie den Herausforderungen, welche Möglichkeiten nützen Sie zur Stressbewältigung?}

Ich bin ja an sich ein positiv denkender Mensch, aber bis Ende des Jahres müssen wir einen Schulentwicklungsplan erstellen - in diesen Zeiten keine leichte Aufgabe. Es ist jetzt alles zeitaufwändiger. Gespräche, die vor der Pandemie in fünf Minuten abgewickelt wurden, dauern nun eine halbe Stunde, vielleicht ist das eine Auswirkung der fehlenden sozialen Kontakte. Positiv ist, dass ich als körperlichen Ausgleich noch mehr als zuvor laufen gehe.

\section{Welche Freuden zur Ablenkung bereiten Sie sich noch?}

Ein gutes Glas Rotwein oder ein rauchiger Single Malt Scotch Whisky am Wochenende können es schon sein und ich habe auch das Glück, dass unsere Mensa offen ist, das ist ein bisschen so wie ins Gasthaus zu gehen. Außerdem habe ich mir einen Oldtimer, einen MGB, gekauft und ich spiele sehr gerne Karambol auf meinem Billardtisch im Keller. So schaffe ich mir einen positiven Ausgleich zur schulischen Arbeit in diesen herausfordernden und schwierigen Zeiten. 